\title{
CORRELATION OF CLINICAL-PATHOLOGICAL VARIABLES WITH THE PATHOLOGIC COMPLETE RESPONSE AFTER NEOADJUVANT CHEMOTHERAPY IN TRIPLE-NEGATIVE BREAST CANCER
}

Gilandira Ivanda Da Costa Soares, Josmara Ximenes Andrade Furtado

'Universidade Federal do Ceará - Fortaleza (CE), Brazil.

Introduction: The triple-negative breast cancer (TNBC) is one of the most aggressive types of breast cancer, corresponding to about $15 \%$ to $20 \%$ of invasive breast tumors. They are those tumors that in immunohistochemistry do not express homone receptors and epidermal growth factor type 2 (cerbB2). This tumor phenotype does not yield many treatment options, beyond standard chemotherapy, and within this context, the evidence of some markers of this type of tumor may contribute to the discovery of more effective types of treatment. Case report and Objectives: The aim of this study was to define predictive and prognostic factors in TNBC that could be related with a pathologic complete response after neoadjuvant chemotherapy treatment. Methods: A descriptive and retrospective study, a case series type, in women with TNBC who had underwent neoadjuvant chemotherapy and surgery at the Mastology Service of Maternidade Escola Assis Chateaubriand - Brazil - from May 2015 to June 2020. A statistical analysis was performed considering the 5\% significance level. Results: From 108 women, only 47 were included in the study, with median age of 49 years (+14 years); about $30(42.6 \%)$ had a family history of breast cancer in first or seconddegree relatives. About $44(93.6 \%)$ cases were classified as invasive ductal tumor and grade II or III; the value of Ki67 greater than $14 \%$ was evidenced in 46 (97.9\%) women and 26 (55.3\%) had clinical stage III. Pathologic complete response to chemotherapy was evidenced in 16 (34\%) cases, partial response in $13(27.7 \%)$ and no response in 18 (38.3\%) cases. The latter cases correspondeded to those who had stable or progression of disease. There was recurrence in 13 (27.7\%) women, about 8 distant metastases, with the lungs as the most frequent site of metastasis followed by the brain. Eleven patients, about $23.4 \%$, died. In the survival analysis of the studied population, the overall survival was 5.6 months and disease-free survival was 19.4 months. No association was observed in the study between the outcome of pathologic complete response to neoadjuvant chemotherapy and anatomopathological characteristics of the tumor. Conclusion: The results of this study did not show statistical significance to determine the possible predictive and prognostic factors for obtaining a complete clinical response to TNBC in a public reference service for the treatment of breast cancer, where there is no genetic signature, PDL1 status or access to differentiated treatment for such a heterogeneous tumor profil. This shows a need for further studies in order to understand this disease and for greater accessibility to high-cost exams and more effective treatments. 